\title{
Obstacle-induced spiral vortex breakdown
}

\author{
Simon Pasche • François Gallaire • Matthieu Dreyer • \\ Mohamed Farhat
}

Received: 30 January 2014/Revised: 30 June 2014 / Accepted: 2 July 2014/Published online: 18 July 2014

(C) Springer-Verlag Berlin Heidelberg 2014

\begin{abstract}
An experimental investigation on vortex breakdown dynamics is performed. An adverse pressure gradient is created along the axis of a wing-tip vortex by introducing a sphere downstream of an elliptical hydrofoil. The instrumentation involves high-speed visualizations with air bubbles used as tracers and 2D Laser Doppler Velocimeter (LDV). Two key parameters are identified and varied to control the onset of vortex breakdown: the swirl number, defined as the maximum azimuthal velocity divided by the free-stream velocity, and the adverse pressure gradient. They were controlled through the incidence angle of the elliptical hydrofoil, the free-stream velocity and the sphere diameter. A single helical breakdown of the vortex was systematically observed over a wide range of experimental parameters. The helical breakdown coiled around the sphere in the direction opposite to the vortex but rotated along the vortex direction. We have observed that the location of vortex breakdown moved upstream as the swirl number or the sphere diameter was increased. LDV measurements were corrected using a reconstruction procedure taking into account the so-called vortex wandering and the size of the LDV measurement volume. This allows us to investigate the spatio-temporal linear stability properties of the flow and demonstrate that the flow transition from columnar to single helical shape is due to a transition from convective to absolute instability.
\end{abstract}

S. Pasche $(\bowtie) \cdot$ M. Dreyer $\cdot$ M. Farhat

Laboratory of Hydraulic Machines, Ecole Polytechnique

Fédérale de Lausanne, 1015 Lausanne, Switzerland

e-mail: simon.pasche@a3.epfl.ch

F. Gallaire

Laboratory of Fluid Mechanics and Instabilities,

Ecole Polytechnique Fédérale de Lausanne,

1015 Lausanne, Switzerland

\section{Introduction}

Vortex breakdown is commonly observed in swirling flows when the swirl number $S$, comparing the respective intensity of the azimuthal and axial velocity components, exceeds a critical value. It occurs in a wide range of applications from leadingedge vortices over delta wings to flame holders in combustion devices. Such occurrences are associated with an abrupt change in the flow topology, often characterized by an internal stagnation point (Hall 1972; Leibovich 1978; Escudier et al. 1982; Escudier 1988; Billant et al. 1998). Besides the axisymmetric breakdown form involving a so-called bubble enclosing a finite region of recirculating fluid, vortex breakdown does also exhibit spiral (or helical) states, which have been observed both experimentally and numerically. The coexistence of axisymmetric and spiral states for the same parameters set was first observed in the famous experiment of Lambourne and Bryer (1961) on delta wings using dye filaments visualizations.

Various hypothesis explaining the existence of spiral vortex breakdowns has been actively discussed over the years. The commonly accepted mechanism that has emerged is that axisymmetric vortex breakdown constitutes an independent phenomenon over which secondary helical disturbances may grow. This scenario, conjectured initially by Escudier et al. (1982), has been confirmed by Ruith et al. (2003), who performed three-dimensional (3D) direct numerical simulations (DNSs) of a Grabovski and Berger vortex (Grabowski and Berger 1976) in a semi-infinite domain, and by Herrada and Fernandez-Feria (2006), who analyzed the dynamics of a Batchelor vortex in a straight pipe using similar techniques. Both studies clearly stress that the early stage of breakdown is axisymmetric, and that the swirl needs to be further increased before this flow pattern is altered by the subsequent development of large-scale spiral waves, wrapped around and behind 
the axisymmetric bubble. Recently, Gallaire et al. (2006) have used the nonlinear front theory on weakly non-parallel flows to investigate the stability properties of the axisymmetric breakdown state computed by Ruith et al. (2003). They have shown that the single helix observed at low swirl can be viewed as the manifestation of a so-called nonlinear steep global mode (see Huerre et al. 2000 for a review), triggered by a transition from convective to absolute local instability in the lee of the axisymmetric bubble. Global linear analysis relaxing this quasiparallel assumption has recently confirmed the interpretation of helical vortex breakdown as a global instability (Meliga et al. 2012; Qadri et al. 2013).

Vortex breakdown is known to be favored by adverse pressure gradients in divergent ducts in case of internal flows (Lopez 1994; Meliga and Gallaire 2011) or as commonly seen in the case of a Francis runner vortex rope, Fig. 1. The study of a vortex impinging on wall, as considered numerically by Herrada et al. (2009), Ortega-Casanova and Fernandez-Feria (2009) and experimentally by Cooper et al. (1993), Alekseenko et al. (2007), can be viewed as the limiting case of strong adverse pressure gradient. We will investigate the situation of weaker and adjustable adverse pressure gradient, considering a vortex impinging on a downstream-located sphere of variable diameter.

\section{Experimental setup}

The experiments were carried out in the EPFL high-speed cavitation tunnel (Avellan et al. 1987). This test facility is a

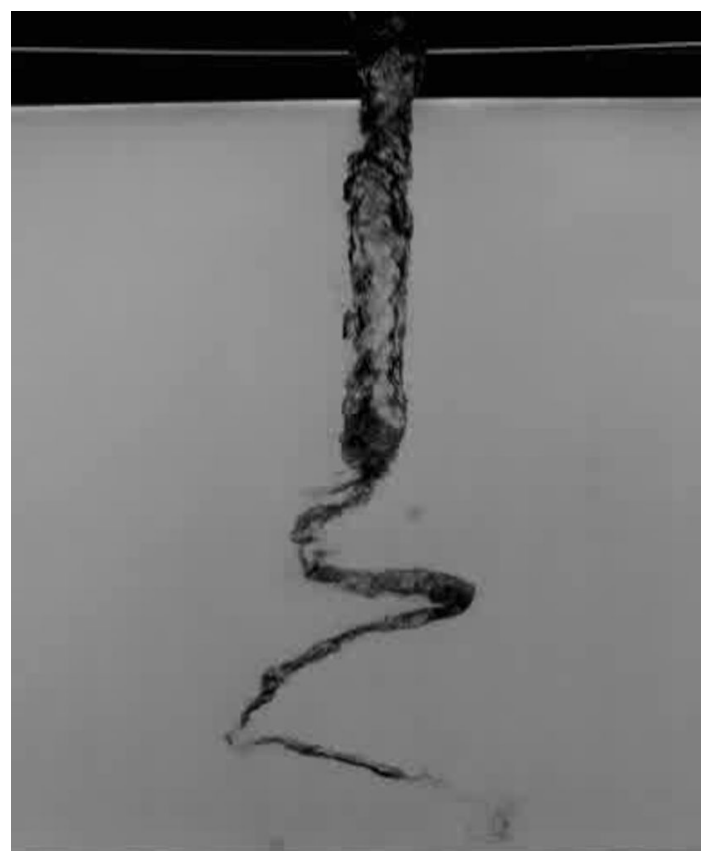

Fig. 1 Photography of a Francis turbine vortex rope, EPFL laboratory for hydraulic machines closed loop with a $150 \times 150 \times 750 \mathrm{~mm}$ square test section. A honeycomb and a 46-1 contraction nozzle are placed upstream of the test section to avoid macroscopic flow rotation and maintain the turbulence intensity below $1 \%$. The static pressure may be increased up to $1.6 \mathrm{MPa}$ and a maximum of $50 \mathrm{~m} / \mathrm{s}$ free-stream velocity may be reached at the inlet of the test section.

The experiment is constituted of an elliptic hydrofoil NACA16020, with a maximum chord length $c=60 \mathrm{~mm}$ and a span $b=87 \mathrm{~mm}$ placed near the inlet of the test section and a spherical obstacle located further downstream to generate an adverse pressure gradient. A visualization of the experiment is displayed in Fig. 2 and a 3D schematic, with an illustration of the vortex trajectory is displayed in Fig. 3. This setup is similar to the test case used by Prothin et al. (2014) and results in the formation of a vortex. The spherical obstacle of diameter $D$ is placed on the axis of the vortex at a distance of $140 \mathrm{~mm}$ from the hydrofoil tip and is mounted on a mechanical device, which allows for its controlled translation in the plane perpendicular to the free-stream velocity so that the vortex axis intersects the sphere at the stagnation point. Additionally, a 0.3 -mm-diameter hole was drilled at the hydrofoil tip to allow for air injection and visualize the vortex flow.

Throughout the study, two systems of coordinates are used. A cartesian coordinate system is chosen so that the $x$ and $z$ axes, respectively, point along the spanwise and the streamwise inverse directions, while the $y$ axis points toward the vertical direction (Fig. 3). The cylindrical system is, on the other hand, aligned on the vortex axis so that both systems of coordinates share the same $z$ axis, originating from the stagnation point and pointing upstream.

The vortex breaks down at a distance denoted $z_{\text {break }}$ from the sphere's upstream pole. The variation of $z_{b r e a k}$ with the free-stream velocity $V_{\infty}$, the sphere diameter $D$ and the hydrofoil incidence angle $\alpha$ was investigated (Table 1).

\section{Flow measurements methods}

Two series of measurements were carried out in this study. First, the vortex breakdown position $z_{\text {break }}$ and the frequency of the spiral $\xi$ were investigated by image processing of highspeed movies. Second, the vortex velocity fields $V_{\theta}(r)$ and $V_{z}(r)$ were measured using LDV techniques.

\subsection{High-speed camera}

The vortex trajectory and its breakdown were visualized using tiny air bubbles injected at the hydrofoil tip and trapped in the vortex core, playing the role of tracers. The visualization equipment is composed of a high-speed camera (Photron SA1.1) set at 80,000 fps and a Xenon flash light, having $100 \mathrm{~J}$ energy and $11 \mathrm{~ms}$ duration. The vortex 
Fig. 2 Bottom view visualization of the obstacleinduced helical vortex breakdown phenomenon revealed by air injection from the wing tip
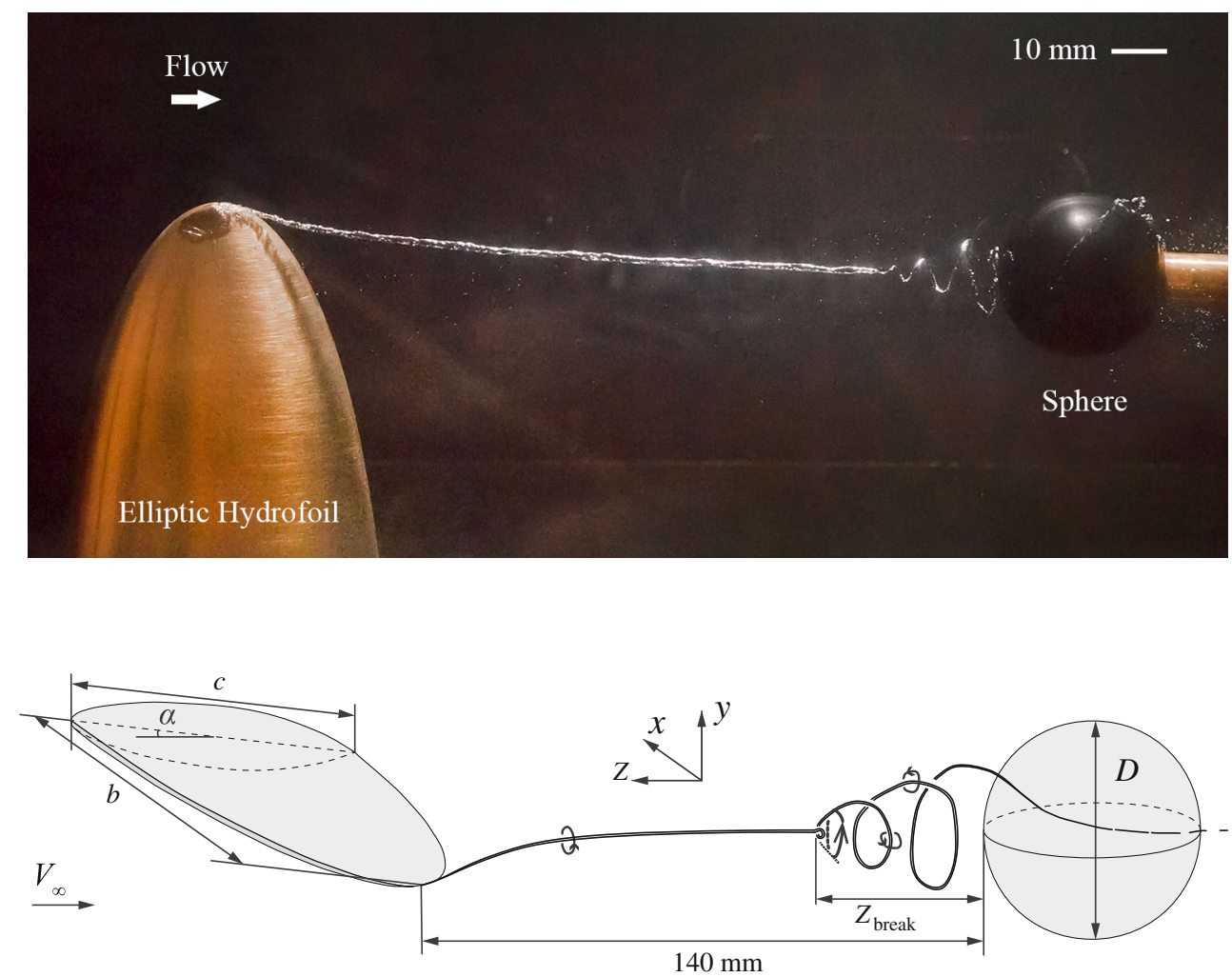

Fig. 3 3D schematic of the experimental setup with an illustration of the vortex trajectory

Table 2 LDV measurement volume sizes

Table 1 Experimental conditions

\begin{tabular}{lllll}
\hline Cases & $\begin{array}{l}\text { Sphere } \\
\text { diameter } \\
\mathrm{D}(\mathrm{mm})\end{array}$ & $\begin{array}{l}\text { Free- } \\
\text { stream } \\
\text { velocity } \\
V_{\infty}(\mathrm{m} / \mathrm{s})\end{array}$ & $\begin{array}{l}\text { Incidence } \\
\text { angles of } \\
\text { hydrofoil for } \\
\text { LDV } \\
\text { measurements }\left(^{\circ}\right)\end{array}$ & $\begin{array}{l}\text { Incidence } \\
\text { angles of } \\
\text { hydrofoil for } \\
\text { Camera } \\
\text { measurements }\left(^{\circ}\right)\end{array}$ \\
\hline$\# 1$ & 20 & 4 & 8,12 & $4-14$ \\
$\# 2$ & 20 & 5.5 & $4,8,12$ & $4-14$ \\
$\# 3$ & 20 & 7 & $4,8,12$ & $4-14$ \\
$\# 4$ & 11.7 & 5.5 & $6,8,12$ & $4-14$ \\
$\# 5$ & 29.8 & 5.5 & $6,8,12$ & $4-14$ \\
\hline
\end{tabular}

breakdown, located at $z_{\text {break }}$, is assumed to initiate where the bubbles start deviating from a straight trajectory. For each test condition listed in Table 1, a series of 5 movies of $11 \mathrm{~ms}$ duration each were recorded and analyzed to identify the mean and standard deviation of the vortex breakdown location and the spiral frequency.

\subsection{Laser doppler velocimetry}

For each of the test conditions listed in Table 1, the axial and azimuthal velocity profiles across the tip vortex were measured using 2 components LDV techniques at 660 and 775

\begin{tabular}{llll}
\hline LDV components & $l_{x}(\mathrm{~mm})$ & $l_{y}(\mathrm{~mm})$ & $l_{z}(\mathrm{~mm})$ \\
\hline \#1 axial & 1.173 & 0.088 & 0.087 \\
\#2 azimuthal & 1.395 & 0.104 & 0.104 \\
\hline
\end{tabular}

nm, Dantec FlowExplorer compact LDA. Our LDV system is composed of a $300 \mathrm{~mW}$ solid state laser source, a beam splitter and an optical probe with $300 \mathrm{~mm}$ focal length. The dimension of the measurement volume is given in Table 2. The seeding particles are some $10 \mu \mathrm{m}$ diameter hollow glass spheres with a density close to the one of water. We assume that these particles follow the liquid perfectly.

The average velocity was measured at 75 positions along the $x$ axis with a refined increment $(0.133 \mathrm{~mm})$ in the vortex core and a coarse increment $(1.33 \mathrm{~mm})$ in the potential zone. The measurements were performed from $x=-16.3 \mathrm{~mm}$ to $x=16.3 \mathrm{~mm}$ (recall that $x=0$ on the vortex axis). The evolution of the velocity profile of the vortex was measured along the $z$ axis, ranging from $z=81$ $\mathrm{mm}$ to $z=1 \mathrm{~mm}$, refined close to the vortex breakdown location. The sampling rate lies between $600 \mathrm{~Hz}$ behind the vortex breakdown and $4000 \mathrm{~Hz}$ in the free-stream zone. The measurement duration was set to $5 \mathrm{~s}$ for all test conditions. 


\section{Vortex model}

In this section, we detail the axisymmetric analytic functions used to fit the velocity profiles. Such functions are introduced since they allow for an accurate determination of the vortex core sizes, the vortex shape parameters, the wake deficit and the wake width. The azimuthal component was fitted using a VM2 model, first introduced by Fabre and Jacquin (2004), which displays a core of radius $a_{c}$ in the solid body rotation with central vorticity $\Omega$, an intermediate region defined by the shape parameter $a_{2} / a_{c}$ where the velocity decays like $r^{-\beta}$ and an external potential region $r>a_{2}$.

$$
V_{\theta}(r)=\frac{\Omega r}{\left[1+\left(r / a_{c}\right)^{4}\right]^{(1+\beta) / 4}\left[1+\left(r / a_{2}\right)^{4}\right]^{(1-\beta) / 4}} .
$$

The wake model for the axial velocity is a pseudo-Voigt profile:

$V_{z}(r)=\eta \cdot V_{\text {Lorentzian }}+(1-\eta) \cdot V_{\text {Gaussian }}$,

where

$$
\begin{aligned}
& V_{\text {Gaussian }}=U_{\infty}+\left(U_{c}-U_{\infty}\right) \cdot e^{-(r / a)^{2}} \\
& V_{\text {Lorentz }}=U_{\infty}+\left(U_{c}-U_{\infty}\right) \cdot \frac{1}{1+(r /(a / 2))^{2}} .
\end{aligned}
$$

This profile models a wake flow with a free-stream velocity $U_{\infty}$ and a velocity deficit given by the weighted sum, $\eta$, of a Gaussian and a Lorentzian with the same width $a$.

As illustrated in Fig. 4 (a) and (b), these families of azimuthal and axial velocity profiles were found to fit well the LDV measurements.

\section{Velocity profile reconstruction}

The time average of the velocity profiles is derived from LDV measurements considering the seeding particles crossing the considered measurement volume. This averaging procedure suffers from two biases. First, the measurement volume size $(1.395 \mathrm{~mm}$ in the azimuthal direction) is of the same order of magnitude as the vortex core. Second, the vortex axis is actually wandering about the measurement point in a random motion. Therefore, the measured velocity profiles are in facts the moving averages of the actual velocity profiles. In order to overcome this issue, we propose in the following a deconvolution method to estimate the effective velocity profiles. The laser intensity forms a Gaussian envelop which can be modeled by a bivariate probability density function:
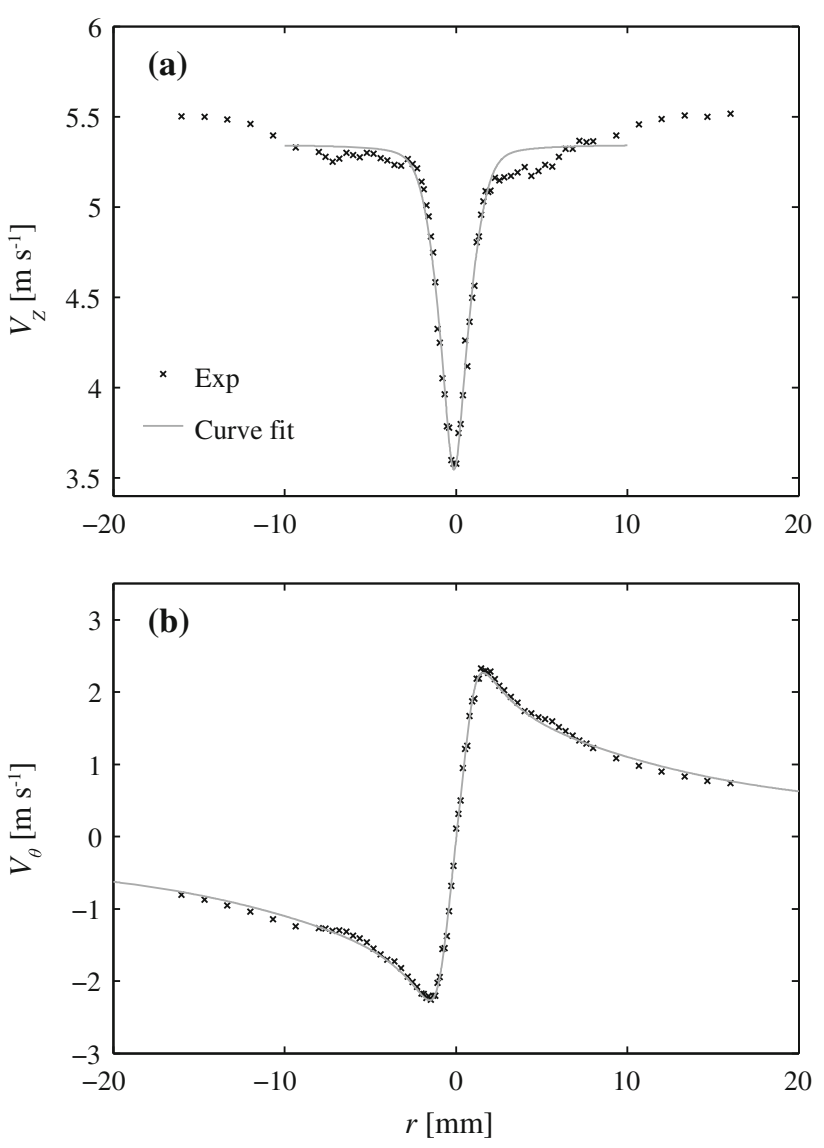

Fig. 4 Experimental velocity profiles (crosses) and fit (solid curve) using VM2 vortex and the pseudo-Voigt model for a free-stream velocity $V_{\infty}=5.5 \mathrm{~m} / \mathrm{s}$, sphere diameter $D=11.7 \mathrm{~mm}$, angle of attack $\alpha=8^{\circ}$ and a $z$-position $z=8.9 \mathrm{~mm}$. Parameters of the velocity profiles: a) $U_{c}=3.55 \mathrm{~m} / \mathrm{s}, a=1.45 \mathrm{~mm}, U_{\infty}=5.35 \mathrm{~m} / \mathrm{s}, \eta=0.52$, b) $\Omega=2.230 \mathrm{rad} / \mathrm{s}, a_{c}=1.26 \mathrm{~mm}, a_{2}=11.34 \mathrm{~mm}, \beta=0.42$

Table 3 function modeling the LDV measurement volume

Variance of the bivariate probability density $\sigma(\mathrm{mm})$ Components

\begin{tabular}{lc}
\hline \#1 axial & $\sigma_{v y}=0.293$, \\
& $\sigma_{v x}=0.022$ \\
\#2 azimuthal & $\sigma_{v y}=0.349$, \\
& $\sigma_{v x}=0.026$
\end{tabular}

$D_{v}=\frac{1}{2 \pi \sigma_{v x} \sigma_{v y}} \exp \left(-\frac{1}{2}\left(\frac{x^{2}}{\sigma_{v x}^{2}}+\frac{y^{2}}{\sigma_{v y}^{2}}\right)\right)$,

where $\sigma_{v x}$ and $\sigma_{v y}$ are the variances of the focussed beam waist in the normal and spanwise direction, respectively. The values of these parameters are equal to $e^{-2}$ times the peak core value, which commonly defines the axes of the elliptical measurement volume (Tables 2, 3).

Consequently, the measured velocity, $U_{p}$, on an infinitesimal ellipse $\left(x_{p}, y_{p}\right)$, may be seen as the weighted average of the real velocity, $U$, associated with the vortex axis 
displacement, $\left(x_{v}, y_{v}\right)$ which is modeled by a bivariate probability density function, $D_{w}$, as proposed by Devenport et al. (1996) and confirmed by Heyes and Hubbard (2003) and Iungo et al. (2009):

$$
U_{p}\left(x_{p}, y_{p}\right)=\int_{-\infty}^{\infty} D_{w}\left(x_{v}, y_{v}\right) U\left(x_{p}-x_{v}, y_{p}-y_{v}\right) \mathrm{d} x_{v} \mathrm{~d} y_{v}
$$

with the vortex wandering defined as

$D_{w}=\frac{1}{2 \pi \sigma_{w}^{2}} \exp \left(-\frac{1}{2 \sigma_{w}^{2}}\left(x^{2}+y^{2}\right)\right)$,

where $\sigma_{w}$ is the wandering amplitude, assumed isotropic. The average velocity field, $U_{m}$, originates from the sum on the infinitesimal ellipses located at $\left(x_{c}, y_{c}\right)$ forming the measurement volume thus writes as the successive convolution of the real velocity profile and the bivariate normal probability densities defined by Eq. 5 and 7, as follows

$$
\begin{aligned}
U_{m}\left(x_{c}, y_{c}\right)= & \int_{-\infty}^{\infty} D_{v}\left(x_{c}-x_{p}, y_{c}-y_{p}\right) \\
& {\left[\int_{-\infty}^{\infty} D_{w}\left(x_{v}, y_{v}\right) U\left(x_{p}-x_{v}, y_{p}-y_{v}\right) \mathrm{d} x_{v} \mathrm{~d} y_{v}\right] } \\
& \mathrm{d} x_{p} \mathrm{~d} y_{p} .
\end{aligned}
$$

Using the following change of variables $x_{t}=x_{c}-x_{p}$, $y_{t}=y_{c}-y_{p}$, and the associativity property of the convolution product, we can write Eq. 8 as:

$U_{m}=\tilde{D} * U$,

with

$$
\begin{aligned}
\tilde{D}= & \frac{1}{2 \pi \sqrt{\sigma_{w x}^{2}+\sigma_{v x}^{2}} \sqrt{\sigma_{w y}^{2}+\sigma_{v y}^{2}}} \\
& \exp \left(-\frac{1}{2}\left(\frac{x^{2}}{\sigma_{w x}{ }^{2}+\sigma_{v x}^{2}}+\frac{y^{2}}{\sigma_{w y}^{2}+\sigma_{v y}^{2}}\right)\right),
\end{aligned}
$$

where the variance is composed by the equivalent measurement volume size, $\sigma_{v}$ and the wandering amplitude, $\sigma_{w}$.

In order to deconvolve the underlying velocity field $U$ from our measurements $U_{m}$ according to Eq. 9, two deconvolution methods proposed by Iungo et al. (2009) and Devenport et al. (1996) may be found in the literature. Iungo's method, which is inspired from blind deconvolution techniques used in astrophysics and medical imaging, requires well-resolved 2D flow fields at each $z$-location. In contrast, Devenport's method is better suited to our 1D measurements $U_{m}(x, y=0)$, which implicitly assume axisymmetry by considering the family of velocity profiles introduced in Sect. 4 as velocity profile candidates instead of a discrete sum of Gaussian functions. In order to determine the seven free shape parameters entirely characterizing the velocity profiles Eqs. $1-4$ as well as the isotropic wandering amplitude $\sigma_{w}$ prevailing at each $z$ station, we use the axial velocity distribution $V_{z}^{m}(x, z)$ and the projection of the tangential velocity $V_{\theta}^{m}(x, z)$ on the vertical $y$ axis, as measured from LDV and iterate on $\sigma_{w}$ until the RMS value of the Reynolds stresses measured on the vortex axis $(r=0)$ matches, within $10^{-3}$ accuracy, the one resulting from the convolution procedure according to

$$
\begin{aligned}
\overline{u v_{m}}\left(x_{c}, y_{c}\right)= & \int_{-\infty}^{\infty} \tilde{D}\left(x_{v}, y_{v}\right) U\left(x_{c}-x_{v}, y_{c}-y_{v}\right) V \\
& \left(x_{c}-x_{v}, y_{c}-y_{v}\right) \mathrm{d} x_{v} \mathrm{~d} y_{v}-U_{m} V_{m} .
\end{aligned}
$$

The effective velocity profiles are thereby determined by least-square optimization: trust region reflective algorithm is used to target a global minimum in a constrained domain. Since the effect of the wandering is to smooth the velocity profiles, which results in an increase of the apparent vortex core, the shape parameters fitted to the measurements can be used as limiting values in the leastsquare algorithm. Deconvolved velocity profiles and relative errors of the method based on synthetic velocity profiles are displayed in Fig 5.

\section{Experimental results}

\subsection{High-speed camera visualizations}

In absence of obstacle, the wing-tip vortex remains slender and does not experience vortex breakdown. In contrast, the vortex systematically escapes from the obstacle through a sudden spiraling motion when a sphere is used. This feature of the flow is particularly reminiscent of vortex breakdown.

The vortex breakdown locations $z_{\text {break }}$ obtained from high-speed visualizations are gathered in Fig. 6a, b. They, respectively, report $z_{\text {break }}$ as a function of the incidence angle $\alpha$ for three different sphere diameters $D$ and three different free-stream velocities $V_{\infty}$. Note that the vortex breakdown location migrates upstream as the incidence angle is increased. This trend is observed in both Fig. 6a, b independently of $D$ and $V_{\infty}$ : it can be ascribed to the swirling intensity of the vortex, as shown on the measured velocity profiles, which increases with the angle of attack and is known to favor vortex breakdown (Leibovich 1978).

Figure 6a, where the free-stream velocity is fixed to 5.5 $\mathrm{m} / \mathrm{s}$ and three sphere diameters were tested, demonstrates that vortex breakdown is favored by larger sphere 

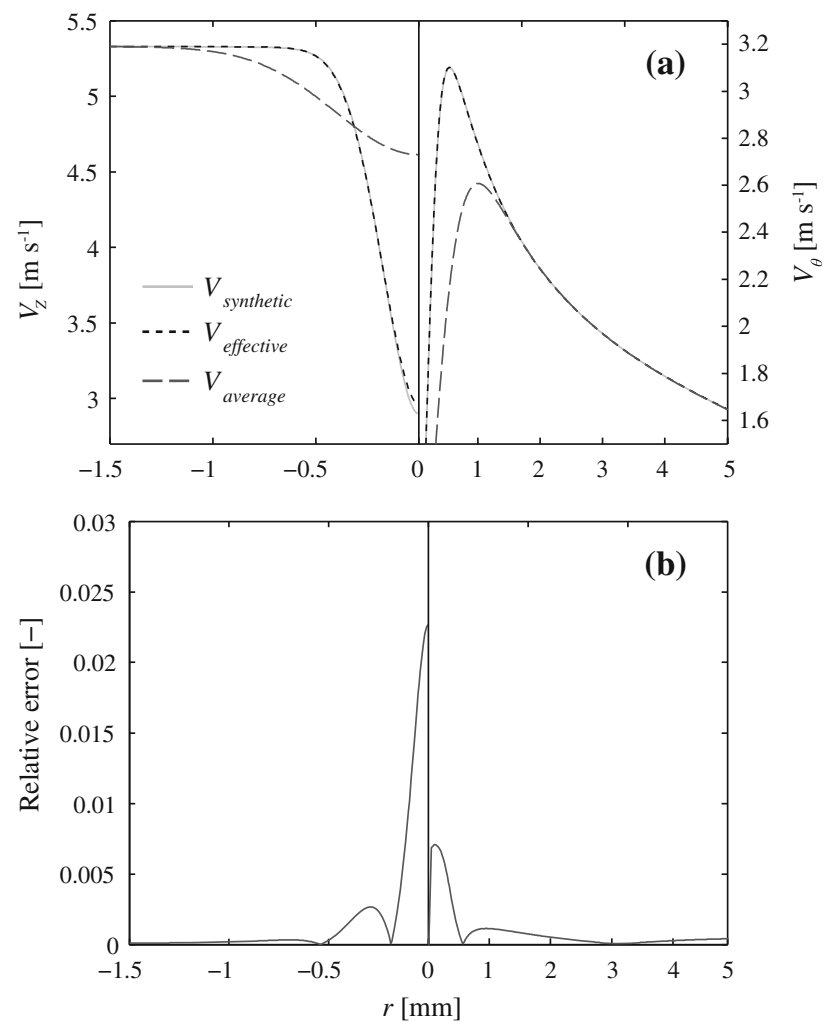

Fig. 5 Velocity profile reconstructions using VM2 model and pseudo-Voigt model (a) and relative errors (b) on synthetic profiles (solid curve). Average velocity calculated by convolution (large dash curve), using Eq. 9, with $\sigma_{w x}=\sigma_{w y}=0.2$ and $\sigma_{v}$ from Table 3. The effective velocity profile(small dash curve), obtained by the deconvolution method based on the average velocity profile

diameters, pointing to a destabilizing influence of the adverse pressure gradient, again in agreement with the literature on vortex breakdown. A rescaling of the vortex breakdown location with the sphere diameter did not allow to collapse the curves, except at small incidence angles.

Figure $6 \mathrm{~b}$, where the sphere diameter is fixed to $D=20 \mathrm{~mm}$ and three free-stream velocity $V_{\infty}=4,5.5,7$ $\mathrm{m} / \mathrm{s}$ are considered, does not allow to clearly identify the influence of the free-stream velocity $V_{\infty}$ on the vortex breakdown location. At low incidence angles, an increase in free-stream velocity favors vortex breakdown but this trend is reversed for larger incidence angles.

\subsection{LDV measurements}

The fits of the raw velocity measurements at different $\mathrm{z}$ positions are systematically processed to extract vortex core sizes, local swirl numbers $S=V_{\theta \max } / V_{\infty}$ and normalized minimum axial velocities $V_{z, r=0} / V_{\infty}$ where $V_{\infty}$ is the free-stream velocity measured at the test section inlet. These key properties are plotted along the $z$ axis and superimposed with the vortex breakdown positions, found
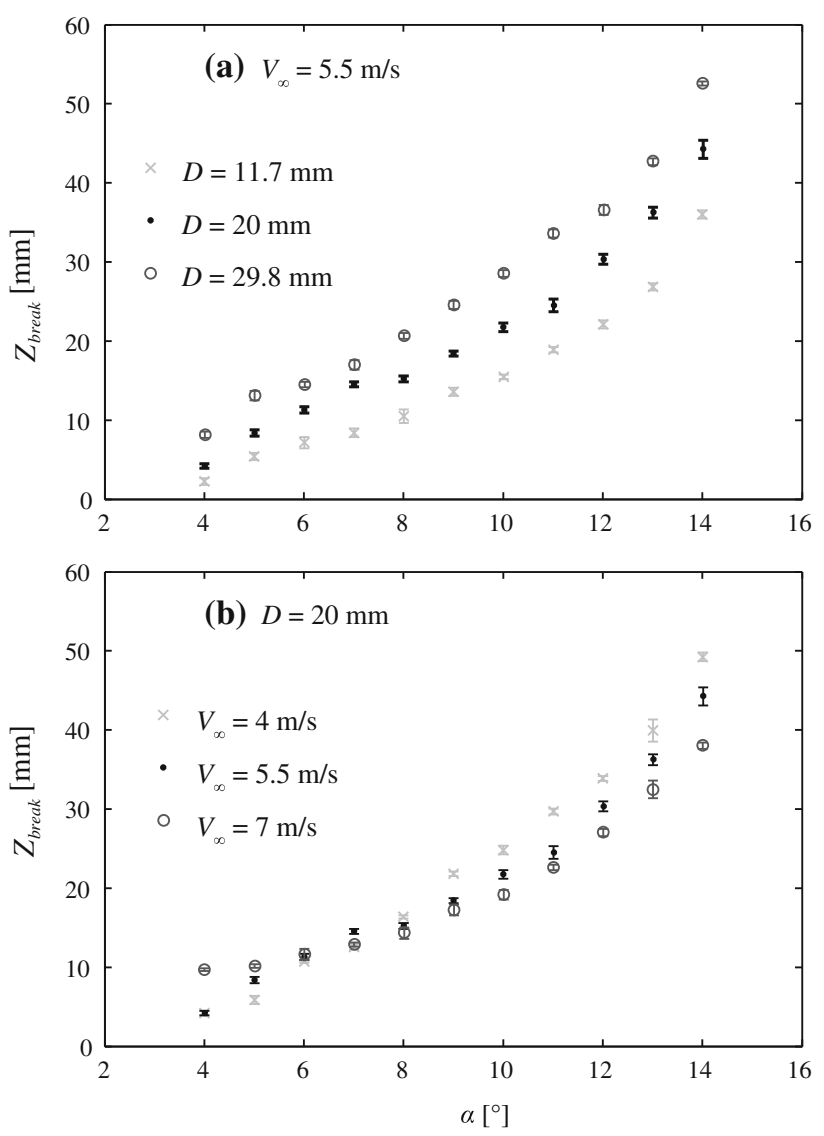

Fig. 6 Vortex breakdown positions, from high-speed visualizations, (a) $D=11.7,20,29.8 \mathrm{~mm}$ and $V_{\infty}=5.5 \mathrm{~m} / \mathrm{s}$, (b) $V_{\infty}=4,5.5,7 \mathrm{~m} / \mathrm{s}$ and $D=20 \mathrm{~mm}$

by image analysis (see Sect. 6.1) and highlighted through full face markers. The sphere diameter $D$ and the incidence angle $\alpha$ are first varied (Figs. 7a, 8a and 9a) for a fixed inflow velocity $V_{\infty}=5.5 \mathrm{~m} / \mathrm{s}$, while the second series of Figs. $7 \mathrm{~b}, 8 \mathrm{~b}$ and $9 \mathrm{~b}$ are obtained for a fixed sphere diameter $D=20 \mathrm{~mm}$ and by varying $V_{\infty}$ and $\alpha$.

The black lines denote the measurements without sphere for a free-stream velocity of $V_{\infty}=5.5 \mathrm{~m} / \mathrm{s}$ and two incidence angles (the black points denote that angle $\alpha=8^{\circ}$ and the black crosses the angle $\alpha=12^{\circ}$ ). These curves highlight the fact that vortex breakdown does not occur in absence of sphere. The vortex remains stable and slender, slowly diffusing by increasing its core size (Fig. 7), reducing its maximal swirling velocity (Fig. 8) and progressively reducing its wake deficit (Fig. 9). This shows that, for the reported incidence angles, the value of the swirl number $\mathrm{S}$ required to trigger vortex breakdown is never attained.

After having being rolled up by the wings, the vortex cores slowly grow almost linearly from $z=81 \mathrm{~mm}$. The vortex core sizes are seen to abruptly expand (Fig. 7) at a location close to the onset of bubble spiraling. As the 
Fig. 7 Evolution of vortex cores, defined at the maximum azimuthal velocity, a $V_{\infty}=5.5$ $\mathrm{m} / \mathrm{s}, D=11.7,20,29.8 \mathrm{~mm}$ and $\alpha=4^{\circ}, 6^{\circ}, 8^{\circ}, 12^{\circ}$, b $D=20$ $\mathrm{mm}, V_{\infty}=4,5.5,7 \mathrm{~m} / \mathrm{s}$ and $\alpha=4^{\circ}, 8^{\circ}, 12^{\circ}$. The vortex breakdown positions from highspeed visualizations are highlighted by the black point
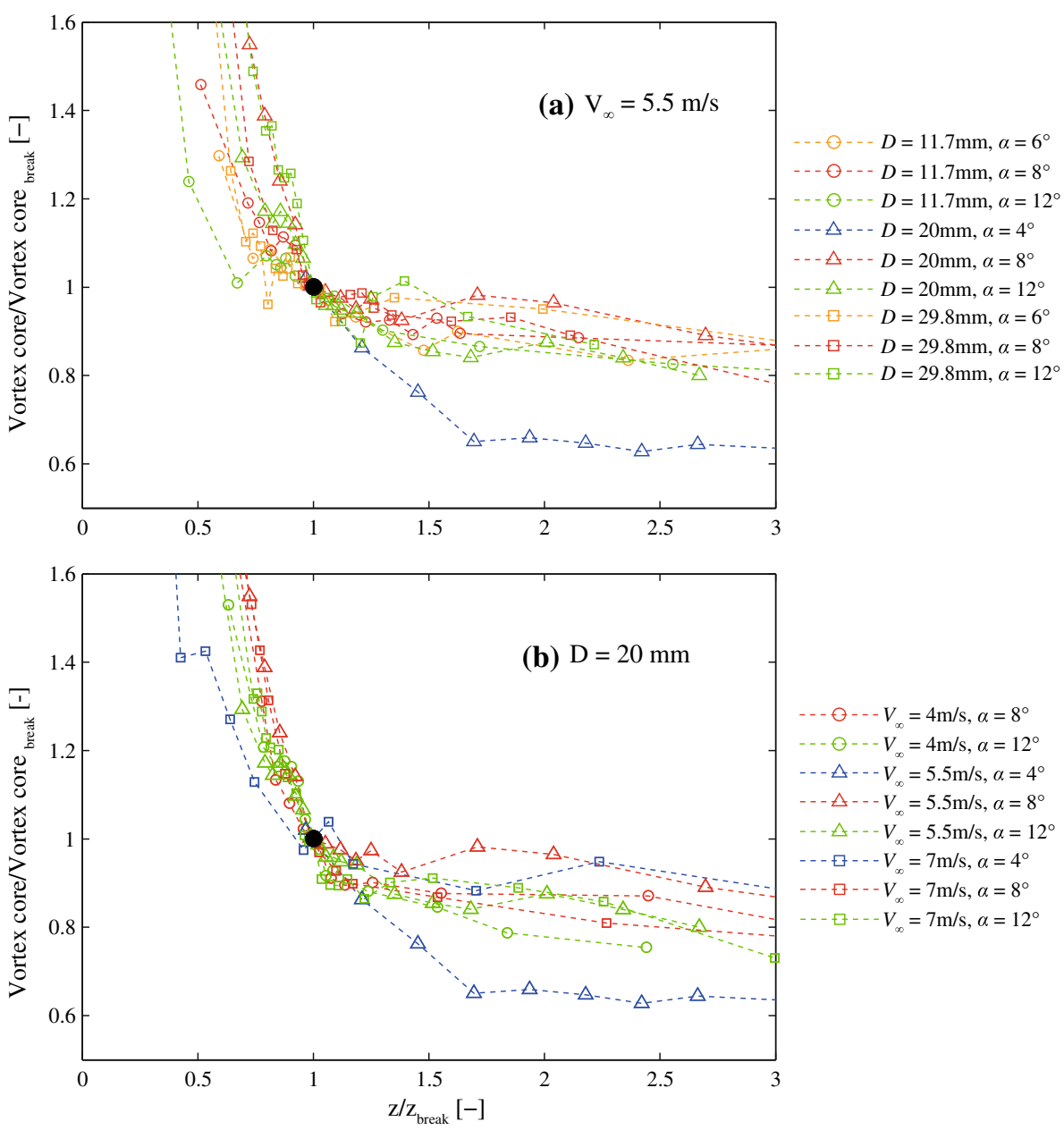

swirling wakes literally burst, the vortex cores further expand, as seen by the post-breakdown slopes of the curves in Fig. 7. The locations of these sudden expansion are seen to be in consistent agreement with the vortex breakdown positions directly measured with the high-speed camera.

Together with this sudden core expansion, the presence of an obstacle causes the maximal tangential velocity (otherwise constantly slowly decaying downstream) to suddenly decrease (Fig. 8). Before breakdown, the presence of the sphere is not felt, as shown by the decay rate which follows the same pace as in absence of obstacle. In complement, Fig.9 demonstrates that the axial velocity field suddenly decelerates as the vortex breakdown location is reached, far ahead from the sphere.

The series of Figs. 7, 8 and 9 therefore show the simultaneous abrupt changes characteristic of vortex breakdown: a sudden expansion of the vortex core, an intense flow retardation and a reduction of the swirling intensity. While vortex breakdown is often associated with a stagnation point and recirculation region, such reversed flow could not be observed in this study. A plausible explanation is the influence of the smoothing effect of the wandering phenomenon and the measurement volume in our direct LDV measurement. We will show later that applying a deconvolution method negative values for the axial velocity are not reached entirely.

Figure $8 \mathrm{a}$ and $\mathrm{b}$ emphasize that the vortex breakdown is favored by a large upstream value of the swirl number. Indeed, for a given sphere diameter or free-stream velocity, vortex breakdown migrates upstream as the swirl number is increased. The collapse of curves in Fig. $8 \mathrm{~b}$ obtained by rescaling the maximum azimuthal velocity by the freestream velocity suggests that the incidence angle is the main control parameter that sets the upstream value of the swirl number and its downstream evolution prior to breakdown. However, the location of breakdown differs for different values of free-stream velocity, pointing out the influence of the detailed velocity profile on the intimate breakdown process, as investigated in Sect. 7 .

The vortex breakdown location is eventually determined by a combination of two main parameters: the upstream value of the swirl, and the sphere diameter. Both tend to favor vortex breakdown: a larger value of the swirl renders the vortex more sensitive to an adverse pressure gradient while a more intense external pressure gradient anticipates vortex breakdown. Obstacle-induced vortex breakdown 
Fig. 8 Evolution of the swirl number $\mathrm{S}$, a $V_{\infty}=5.5 \mathrm{~m} / \mathrm{s}, D=$ $11.7,20,29.8 \mathrm{~mm}$ and $\alpha=4^{\circ}, 6^{\circ}, 8^{\circ}, 12^{\circ}$, b) $D=20$ $\mathrm{mm}, V_{\infty}=4,5.5,7 \mathrm{~m} / \mathrm{s}$ and $\alpha=4^{\circ}, 8^{\circ}, 12^{\circ}$. The vortex breakdown positions from highspeed visualizations are highlighted by the full face markers
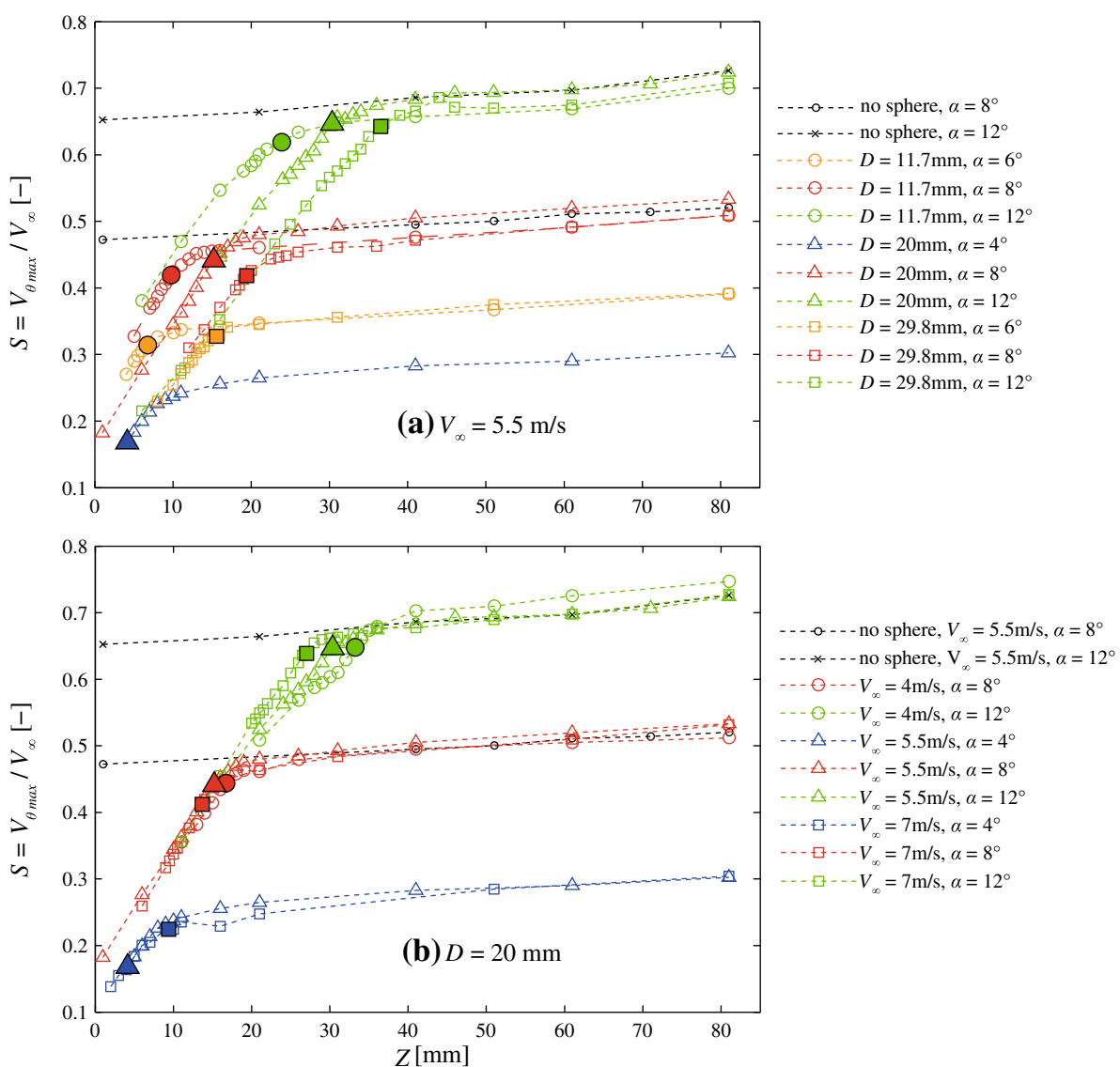

cannot be explained by the pure stagnation flow induced by the obstacle, nor the pure diffusion of the vortex. It is the combination of both effects through a strong coupling of the external pressure gradient with the internal balance between pressure gradient and centrifugal force that conspires to trigger vortex breakdown, in analogy to the strong viscous-inviscid interaction in separated boundary layers.

This strong coupling is readily illustrated in Fig. 10a, where the minimum axial velocity pertaining to potential flow around a sphere, given by Eq. (12),

$V_{\min }(z)=V_{\infty}\left(1-\frac{R^{3}}{z^{3}}\right)$,

is superimposed with experimental results in presence of a wing-tip vortex. In addition, the adverse pressure gradient deduced from this potential solution is illustrated in Fig. 10b. Only a weak adverse pressure gradient is needed to modify the flow pattern.

\section{Local stability analysis}

A spatio-temporal local stability analysis of the effective velocity fields, prevailing at different locations along the $z$ axis are determined under the quasi-parallel assumption. The dispersion relation is analyzed, assuming a normal mode expansion $\exp (i(k x-\omega t))$, where $\omega$ and $k$ denote the complex frequency and the complex wavenumber, respectively. The asymptotic impulse response is actually governed by the saddle point $\omega_{i}^{0}(z)$ of the complex dispersion relation $\omega(k)$ in the complex $k$-plane that has the highest imaginary part and is a valid saddle point (Huerre et al. 1998 for details). The sign of the imaginary part of this point $\omega_{i}^{0}(z)$ determines if the flow is locally absolutely unstable $\left(\omega_{i}^{0}(z)>0\right)$ or convectively unstable $\left(\omega_{i}^{0}(z)<0\right)$. The weakly non-parallel but strongly nonlinear theory of Pier et al. (2001) suggests that, if there exists a station $z_{c / a}$ where $\omega_{i}^{0}(z)$ vanishes and where the flow changes from convectively unstable for $z<z_{c / a}$ to absolutely unstable for $z>z_{c / a}$, a nonlinear global mode may be triggered with a front located at $z_{c / a}$. The global mode then inherits the real absolute frequency $\omega_{0}\left(z_{c / a}\right)$ as global frequency $\omega_{g}{ }^{N L}$.

For the present purpose of analyzing axisymmetric swirling jets and wakes, the Navier-Stokes equations in cylindrical coordinates are linearized, expanded in Fourier series with axial wavenumber $k$ along the $z$-axis and azimuthal wavenumber $m$ along the $\theta$-axis. 
Fig. 9 Evolution of the minimum axial velocities scaled by the free-stream velocity $V_{z, r=0} / V_{\infty}$, (a) $V_{\infty}=5.5 \mathrm{~m} / \mathrm{s}$, $D=11.7,20,29.8 \mathrm{~mm}$ and $\alpha=4^{\circ}, 6^{\circ}, 8^{\circ}, 12^{\circ}$, (b) $D=20$ $\mathrm{mm}, V_{\infty}=4,5.5,7 \mathrm{~m} / \mathrm{s}$ and $\alpha=4^{\circ}, 8^{\circ}, 12^{\circ}$. The vortex breakdown positions from highspeed visualizations are highlighted by the full face markers
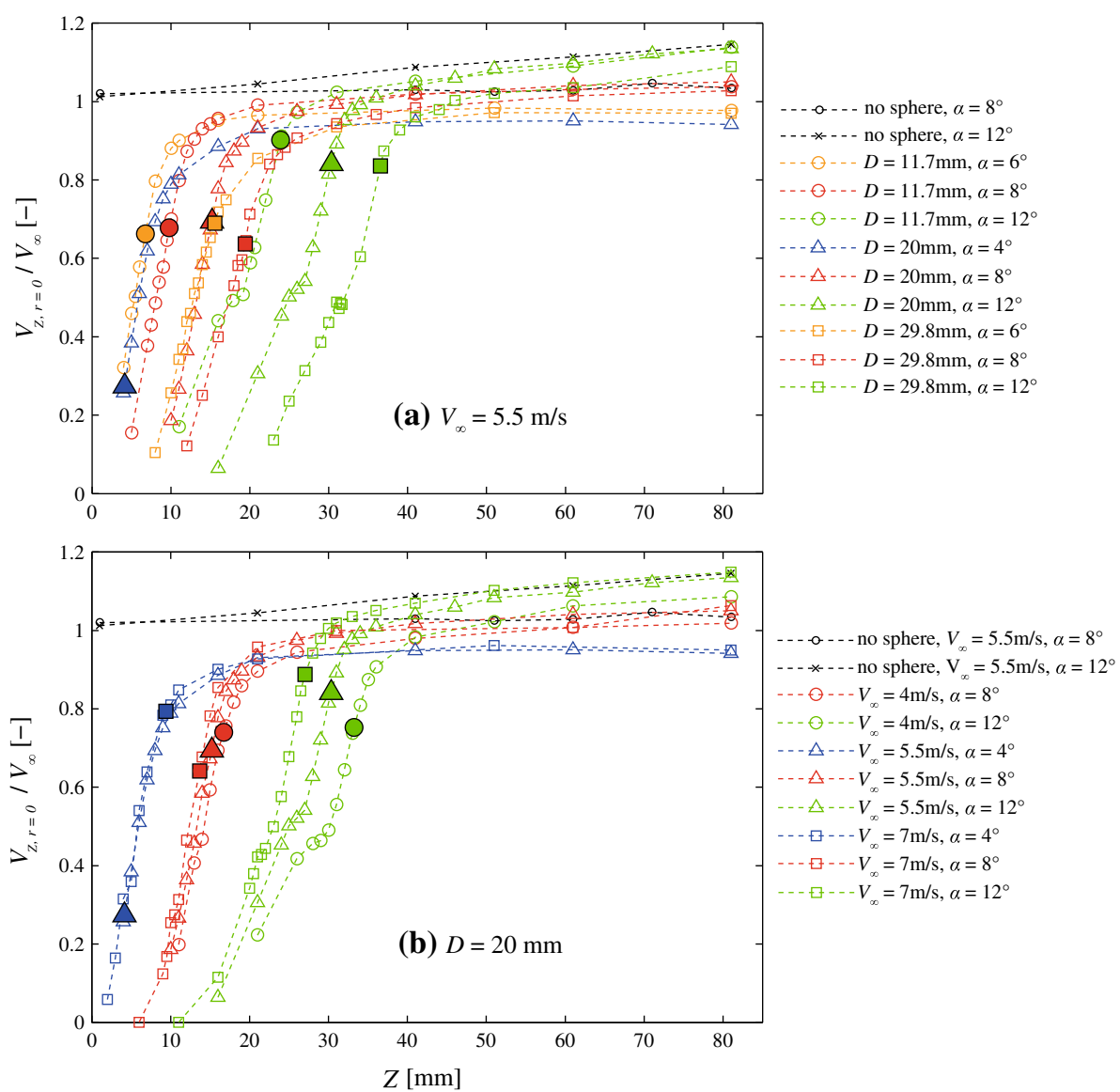

$(\boldsymbol{v}, p)(r, \theta, z, t)=(\boldsymbol{v}, p)(r) \exp (i(k z+m \theta-\omega t))$.

The result is a one-dimensional, linearized system of four equations. The stability analysis based on the code developed by Antkowiak and Brancher (2004) discretizes this system by Chebyshev spectral collocation method.

The absolute/convective properties of the flow are defined by the value of the pulsation $\omega_{i}^{0}$ of the saddle point in the complex $k$ plane, verifying $\frac{d \omega}{d k}=0$ (Huerre et al. 1998). Once a valid saddle point $\omega_{0}\left(z_{0}\right)$ has been determined, it is tracked by a continuation procedure for each base flow along the $z$ axis, using base flows characterized by 8 parameters (the vortex core size $a_{c}$, shape parameters $\alpha, a_{2}$, core vorticity $\Omega$, wake width $a$, Reynolds number $R e=\Omega a_{c}^{2} / v$, minimum axial velocity $U_{c}$, weight $\eta$ ) that are interpolated linearly between the LDV measurement positions. The exact location $k_{0}$ of the saddle point is determined using the method of Deissler (1987), and the fact that the function $\omega(k)$ locally admits a quadratic Taylor expansion around $k_{0}$, which allows to devise a simple convergent iterative scheme.

Streamwise spatio-temporal linear stability analysis was performed on the effective velocity profiles used as base flow for the azimuthal wavenumber $m=1$ and $m=-1$. The maximum temporal growth rate $\omega_{\text {imax }}$ was positive only for the mode $m=-1$. This mode expresses that the vortex is coiling in the opposite direction and temporally rotating in the same direction as the vortex, in agreement with our experimental observations, as seen in Fig. 3.

A typical example is provided in Fig. 11, which shows the results of the spatio-temporal linear stability analysis: the maximum temporal growth rate of the deconvolved velocity profiles $\omega_{i}^{\max }$ and the absolute growth rate $\omega_{i}^{0}$ of the effective velocity field. If we had attempted to determine similar properties from the raw LDV measurements, the flow would have turned linearly stable for almost all streamwise stations upstream of vortex breakdown.

In contrast, the transitions from convectively unstable in the upstream region of the flow to absolutely unstable in the downstream region of the flow take place at locations which correspond well with the measured vortex breakdown positions (see Table 4). At these transitions, the effective axial velocities do not reach negative values and no recirculation region is observed. This suggests that no stagnation point is associated with the vortex breakdown in this experiment and that a direct transition from columnar to single helical flow occurs. In addition, the predicted frequencies of the $m=-1$ spiral that can be argued to be selected by the value of $\omega_{r}^{0}$ at the convective to absolute 

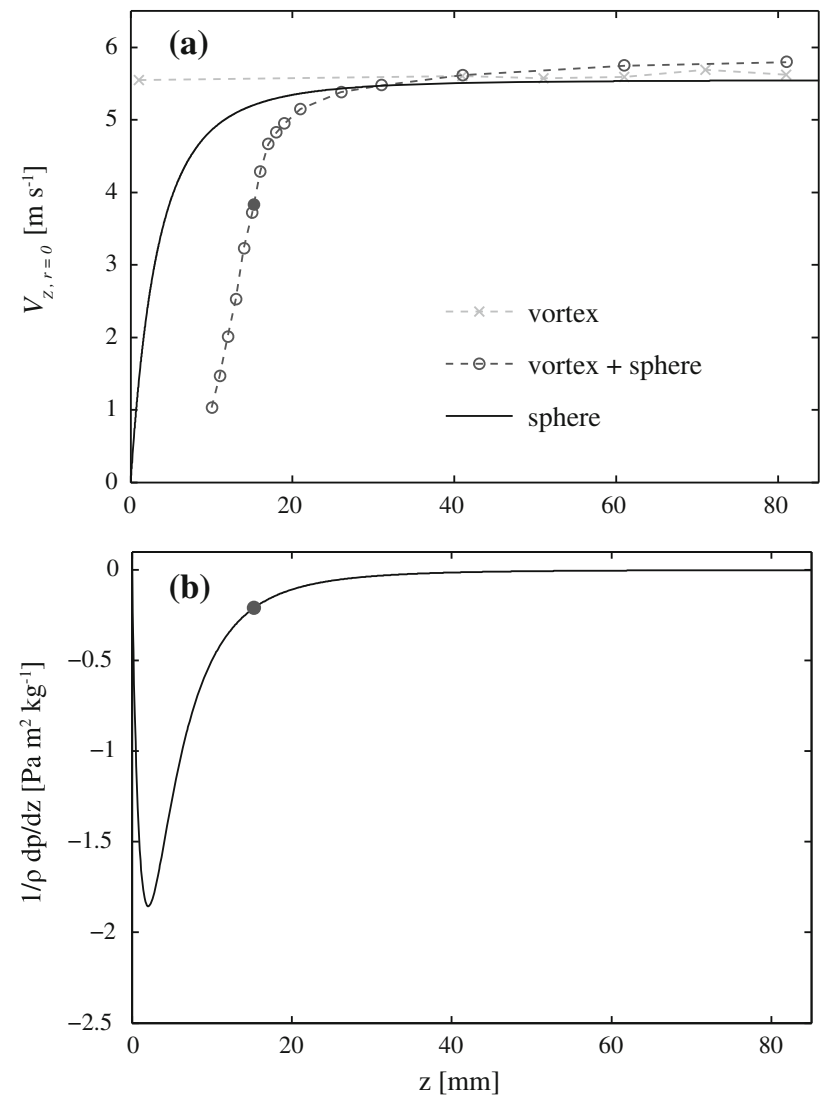

Fig. 10 Evolution of minimum axial velocities a for the potential flow field induced by the sphere of diameter $D=20 \mathrm{~mm}$ (solid curve) and for the vortex with (circles) and without (crosses) the downstream sphere. Evolution of the axial pressure gradient $\mathbf{b}$ for the potential flow around the same sphere, case: $V_{\infty}=5.5 \mathrm{~m} / \mathrm{s}, D=20 \mathrm{~mm}$ and $\alpha=8^{\circ}$. The vortex breakdown positions from high-speed visualizations are highlighted by the full face markers

transition points (Pier et al. 2001), are seen to be in reasonable qualitative agreement with the frequencies $\xi$ that could be measured by image analysis, as seen in Table 4 .

\section{Conclusion}

An original experiment was performed to investigate the vortex breakdown dynamics in flowing water in EPFL high-speed hydrodynamic tunnel. A wing-tip vortex is generated by an elliptical hydrofoil, placed in the test section inlet while a downstream sphere is used to impose adverse pressure gradient along the vortex axis. We have performed high-speed visualizations as well as axial and azimuthal velocity measurements with 2D Laser Doppler Velocimetery (LDV). The swirl number, i.e., maximum azimuthal velocity divided by the free-stream velocity, along with the adverse pressure gradient are the key parameters for the vortex breakdown phenomenon. They
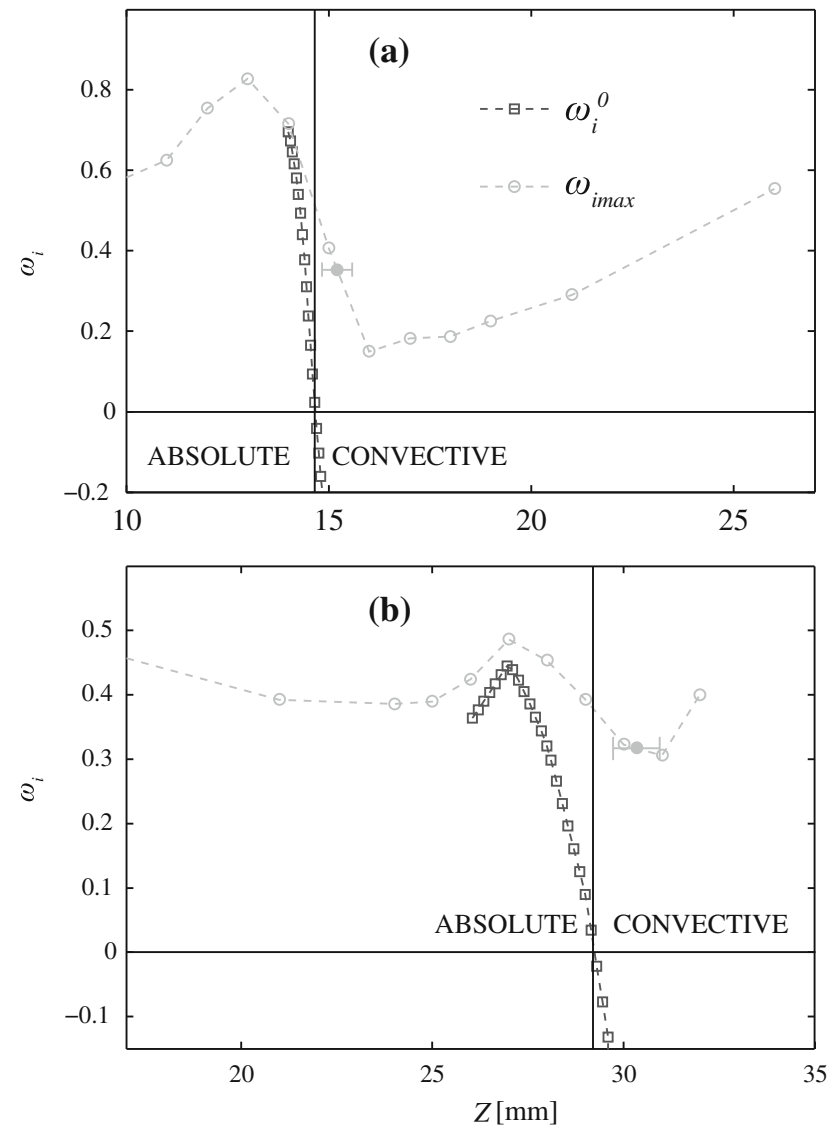

Fig. 11 Evolution of the growth rates $\omega_{i}^{0}$ and $\omega_{\text {imax }}$ for the effective velocity field for an azimuthal wavenumber $m=-1$. The vortex breakdown positions from high-speed visualizations are highlighted by the full face markers, (a) $V_{\infty}=5.5 \mathrm{~m} / \mathrm{s}, D=20 \mathrm{~mm}$ and $\alpha=8^{\circ}$, (b) $V_{\infty}=5.5 \mathrm{~m} / \mathrm{s}, D=20 \mathrm{~mm}$ and $\alpha=12^{\circ}$

were controlled through the hydrofoil incidence angle, the upstream velocity and the sphere diameter. Without obstacle, the vortex remained slender with a slow diffusion. We have systematically observed for all tested operating conditions and sphere diameters a single helical breakdown of the vortex, which coils around the sphere in the opposite direction and rotates in the same direction as the vortex. A reconstruction procedure of the velocity profiles was introduced to correct the space and time averaging effects, which are due to the vortex wandering and the LDV measurement volume size. This allowed us to investigate the spatio-temporal linear stability properties of the flow and demonstrate that the flow transition from columnar to single helical shape is due to a transition from convective to absolute instability. In our experiment, we have not observed any stagnation point at the vortex breakdown position. This suggests that in presence of weak adverse pressure gradient, this flow transition is direct, bypassing the axisymmetric recirculation bubble, in contrast with the case of spiral vortex breakdown identified as a secondary 
Table 4 Comparison of the vortex breakdown positions and the spiral frequencies obtained by high-speed visualizations $\xi$ and by local linear stability analysis for different cases

\begin{tabular}{|c|c|c|c|c|c|c|}
\hline \multirow[b]{2}{*}{ Case } & \multicolumn{3}{|l|}{$z_{\text {break }}(\mathrm{mm})$} & \multicolumn{3}{|c|}{ Frequency $(\mathrm{Hz})$} \\
\hline & $\# 1$ & $\# 2$ & $\# 3$ & $\# 1$ & $\# 2$ & $\# 3$ \\
\hline Experimental & $30.3 \pm 0.6$ & $30.3 \pm 0.6$ & $19.4 \pm 0.3$ & $1,055 \pm 274$ & $1,175 \pm 130$ & $1,145 \pm 260$ \\
\hline $\begin{array}{l}\text { Stability } \\
\text { analysis }\end{array}$ & 14.6 & 29.2 & 19.6 & 1,148 & 1,150 & 878 \\
\hline
\end{tabular}

Case \#1: $D=20 \mathrm{~mm}, V_{\infty}=5.5 \mathrm{~m} / \mathrm{s}, \alpha=8^{\circ}$. Case \#2: $D=20 \mathrm{~mm}, V_{\infty}=5.5 \mathrm{~m} / \mathrm{s}, \alpha=12^{\circ}$. Case \#3: $D=29.8 \mathrm{~mm}, V_{\infty}=5.5 \mathrm{~m} / \mathrm{s}, \alpha=8^{\circ}$

disturbance growing over the axisymmetric vortex breakdown (Gallaire et al. 2006). Higher swirl numbers and larger spheres were observed to induce an upstream migration of the vortex breakdown position. The exact spatial location of vortex breakdown is dictated by a strong coupling between the external pressure gradient caused by the downstream-located sphere and the internal balance between pressure gradient and centrifugal force in the core of the wing-tip vortex. A natural continuation of this experimental work is to study the fluid structure interaction of a swirling jet/wake impinging on a spring-mounted obstacle.

\section{References}

Alekseenko S, Bilsky A, Dublin V, Markovich D (2007) Experimental study of an impinging jet with different swirl rates. Int J Heat Fluid Flow 28:1340

Antkowiak A, Brancher P (2004) Transient energy growth for the lamb-oseen vortex. Phys Fluids 16(1):L1-L4

Avellan F, Herre P, Ryhming IL (1987) A new high speed cavitation tunnel. ASME 57:49-60

Billant P, Chomaz JM, Huerre P (1998) Experimental study of vortex breakdown in swirling jets. J Fluid Mech 376:183-219

Cooper D, Jackson D, Launder B, Liao G (1993) Impinging jet studies for turbulence model assessment-I. Flow field experiments. Int J Heat Mass Transfer 36(10):2675-2684

Deissler RJ (1987) The convective nature of instability in plane poiseuille flow. Phys Fluids 30:2303-2305

Devenport WJ, Rife MC, Liapis SI, Follin GJ (1996) The structure and development of a wing-tip vortex. J Fluid Mech 312:67-106

Escudier M, Bornstein J, Maxworthy T (1982) The dynamics of confined vortices. Proc R Soc Lond Ser A 382:335-360

Escudier MP (1988) Vortex breakdown: observations and explanations. Prog Aerospace Sci 25:189-229

Fabre D, Jacquin L (2004) Short-wave cooperative instabilities in representative aircraft vortices. Phys Fluids 16:1366

Gallaire F, Ruith M, Meiburg E, Chomaz JM, Huerre P (2006) Spiral vortex breakdown as global mode. J Fluid Mech 549:71-80

Grabowski W, Berger S (1976) Solutions of the navier-stokes equations for vortex breakdown as a global mode. J Fluid Mech 75:71-80

Hall M (1972) Vortex breakdown. Ann Rev Fluid Mech 4:195-218
Herrada MA, Fernandez-Feria R (2006) On the development of threedimensional vortex breakdown in cylindrical regions. Phys Fluid 18(084105):1-15

Herrada MA, Pino CD, Ortega-Casanova J (2009) Confined swirling jet impingement on a flat plate at moderate Reynolds numbers. Phys Fluids 21(013601):1-9

Heyes A, Hubbard S, Marquis A, DS, (2003) On the roll-up of a trailing vortex sheet in the very near field. Proc Inst Mech Eng G J Aerospace Eng 217:217-269

Huerre P, Rossi M, Godreche C, Manneville P (1998) Hydrodynamic instabilities in open flows. Cambridge University Press, In Hydrodynamic and Nonlinear Instabilities

Huerre P, Batchelor G, Moffat H, Worster M (2000) Open shear flow instabilities. In Perspectives in fluid dynamics, Cambridge University Press

Iungo GV, Skinner P, Buresti G (2009) Correction of wandering smoothing effects on static measurements of a wing-tip vortex. Exp Fluids 46:435-452

Lambourne NC, Bryer DW (1961) The bursting of leading-edge vortices: some observations and discussion of the phenomenon. Aeronautical Research Council, Report \& Memoranda R \& M 3282:1-35

Leibovich S (1978) The structure of vortex breakdown. Ann Rev Fluid Mech 10:221-246

Lopez J (1994) On the bifurcation structure of axisymmetric vortex breakdown in a constricted pipe. Phys Fluids 6:3683-3693

Meliga P, Gallaire F (2011) Control of axisymmetric vortex breakdown in a constricted pipe: nonlinear steady states and weakly nonlinear asymptotic expansions. Phys Fluids 23:1-23

Meliga P, Gallaire F, Chomaz JM (2012) A weakly nonlinear mechanism for mode selection in swirling jets. J Fluid Mech 699:216-262

Ortega-Casanova J, Fernandez-Feria R (2009) Three-dimensional transitions in a swirling jet impinging against a solid wall at moderate reynolds numbers. J Fluid Mech 21(034107):1-9

Pier B, Huerre P, Chomaz JM (2001) Nonlinear self-sustained structures and fronts in spatially developing wake flows. J Fluid Mech 435:147-174

Qadri UA, Mistry D, Juniper MP (2013) Structural sensitivity of spiral vortex breakdown. J Fluid Mech 720:558-581

Ruith MR, Chen P, Meiburg E, Maxworthy T (2003) Threedimensional vortex breakdown in swirling jets and wakes: direct numerical simulation. J Fluid Mech 486:331-378

Prothin S, Djeridi H, Billard J-Y (2014) Coherent and turbulent process analysis of the effects of a longitudinal vortex on boundary layer detachment on a NACA0015 foil. J Fluids Struct 47:2-20 